\title{
Modelling Light Curves of Systems with Non-Circular Accretion Disks: KU Cyg
}

\author{
S. Zola ${ }^{1,2}$ and T. Szymanski ${ }^{1}$ \\ ${ }^{1}$ Astronomical Observatory, Jagiellonian University, \\ ul. Orla 171, PL-30-244, Krakow, Poland \\ email: szola@oa.uj.edu.pl \\ ${ }^{2}$ Mt. Suhora Observatory, Pedagogical University, \\ ul. Podchorazych 2, PL-30-084 Krakow, Poland \\ email: green@oa.uj.edu.pl
}

\begin{abstract}
We present a code which can be used to simulate the light curve of a binary system harbouring a non-circular accretion disk, and show that such a system geometry can produce light curves with asymmetric minima and different heights of the maxima. We have applied this code to check for the possible existence of a non-circular accretion disk, as suggested by Smak \& Plavec (1997), in the Algol-type binary KU Cyg. We compare the best solutions for two sets of multicolour light curves, obtained within circular and non-circular disk models.
\end{abstract}

Keywords. binaries:eclipsing, accretion disks, stars: individual (KU Cyg)

\section{Non-circular disk model and its effects on the light curve}

If there is an accretion disk in a binary system, the simplest approach to simulate a theoretical light curve is to assume that the disk is circular in shape. Such a model was described by Zola (1992), and applied to solve the light curves of several long period Algols with accretion disks. In this work, we describe a modification to that code which accounts for non-circular disk effects. The properties of the model are essentially the same: it is based on the Wilson-Devinney code, the system configuration is semi-detached and the disk surrounds the star eclipsed at phase 0 . The disk is optically thick, and its geometrical thickness grows linearly with radius. The new modification requires two additional parameters to describe how the disk is "disturbed" from the circular shape: one to define the elliptical shape, while the other to define the disk tilt. The code accounts for geometrical effects arising from the presence of the disk: eclipses of the mass-losing star by the disk, partial or total obscuration of the mass-gaining star, and self-obscuration of the disk at high inclinations. In our model, the disk becomes thicker for parts of the disk with larger radii. This property causes a range of effects in the light curve which are absent when a circular accretion disk is considered.

To display the effects, we have chosen two sets of parameters and created theoretical light curves: one for a high inclination system $\left(i=89^{\circ}\right)$ and the other for a lower $\left(i=85^{\circ}\right)$ inclination system. The angle $\beta$, defining the disk thickness, has been chosen to be $5^{\circ}$ for the lower inclination and $20^{\circ}$ for the higher one. While the system mass ratio, temperatures of the components, and the outer disk temperature were the same for both sets of data, the radius of the primary, mass-gaining star has been chosen such that it was hidden in the disk for the case of the higher inclination system and partly obscured (part of the upper hemisphere visible) for the lower inclination one. Both light curves exhibit a difference in level of the maxima. This is entirely due to the non-circular shape of the disk, which is also responsible for a non-uniform light contribution from the disk 
outside of the phases when the disk is eclipsed. An additional feature is the asymmetry of the secondary minimum. Both effects are caused by the changing disk surface projection alone, in the case of high inclination system, and the combined effects of the projection (light rise) and the obscuration of the mass gaining star by the disk (decrease of light at the same phase).

\section{Application of the model to KU Cyg}

KU Cyg is a long period, Algol-type system showing double-peaked Balmer emission lines (Olson 1988, Olson et al. 1995) visible throughout the entire orbital period. The analysis of photometric data with the W-D code (Wilson 1979) have been done by Olson et al. (1995) and by Zola (1992), but using a code accounting for the presence of a disk. There is some controversy about the disk shape: Nguyen and Etzel (1999) found it to be circular while Smak and Plavec (1997), based on their analysis of a single $\mathrm{H}_{\alpha}$ profile, argued for a significant distortion from the circular shape.

This work is aimed at checking the model of Smak and Plavec (1997), applying the code described in previous sections. We performed light curve modelling of two available sets of data: Olson's vbyI and more recent, BVRI data collected at Mt. Suhora Observatory. The Monte Carlo search method (Zola et al. 2004) was applied to find the best solution within the circular and non-circular disk models, separately for both sets of light curves. Two crucial parameters were fixed at the values derived by Smak and Plavec: the system inclination: $i=86^{\circ}$ and the mass ratio $q=0.13$. In addition, we set the secondary temperature to $3750 \mathrm{~K}$, appropriate for its spectral type.

\section{Results}

The light curve solutions of the two available sets of KU Cyg photometric light curves within a circular disk model resulted in a worse fit and very different parameters for the primary star. The non-circular disk model solutions turned out to fit better and to be consistent with regard to the parameters of the mass-gaining component. We derived 9400-10100 K for its temperature and $0.052-0.053$ for its size, in separation units. We found slightly different disk properties (disk size and geometrical thickness) at the epochs when the two sets of data were gathered. The solution of Olson's data resulted in a disk somewhat bigger and thicker but less eccentric than that derived from data taken at Mt. Suhora. However, the position angle values were found to be very different: $336^{\circ}$ versus $157^{\circ}$ respectively for the Olson and Mt. Suhora data. Our results derived from photometric data seem to support the Smak and Plavec (1997) prediction that tidal effects, similar to those observed in cataclysmic variables (Osaki 1989), would cause the accretion disk in KU Cyg to be unstable and precess with a timescale of a few years.

\section{References}

Nguyen Q. T. \& Etzel P. B. 1999, AAS, 195,7613

Olson E. C. 1988, AJ, 96, 1430

Osaki Y. 1989, PASJ, 41, 1001

Olson E. C., Etzel P. B., \& Dewey M. R. 1995, AJ, 110, 2378

Smak J. I. \& Plavec M. J. 1997, AcA, 47, 345

Wilson R. E. 1979, ApJ, 234, 1054

Zola S. 1992, AcA, 42, 355

Zola S., Rucinski S. M., Baran A., et al., 2004, AcA, 54, 299 